\title{
Multiagent Application in Mobile Environments to Data Collection in Park Zones
}

\author{
María Navarro, Fernando de la Prieta, Gabriel Villarrubia, \\ and Mohd Saberi Mohamad \\ Department of Computer Science, University of Salamanca Plaza de la Merced s/n, \\ 37008, Salamanca, Spain \\ \{mar90ali94,fer,gvg\}@usal.es, mohd.saberi@gmail.com
}

\begin{abstract}
This paper presents an application of automatic parking which assists users. For this, recognition techniques are used, such as the algorithm to recognize a license plate from a photograph or the use of NFC protocol to identify an object in the environment. As well, multiagent system integration will be necessary in order to communicate the different devices and data providers involved in this system. We obtain a system that assists the users in his payments and vehicle identification in an efficient way.
\end{abstract}

Keywords: Virtual organizations, NFC protocol, OCR techniques, neural nets, morphologic operations, agent system, clustering.

\section{Introduction}

Nowadays, the car is essential for our lives, not only for travel but also for everyday tasks like going to work or shopping. Due to this increased use of vehicles, there are time constraints and parking space. In cities, there are zones, called blue and green zones. Here, the payment system is based on a machine in which a ticked is obtained. This ticket depends on the time you have paid for, but it is a pre-payment form. There exists a guard, who supervises that every user is paying for the parking service. This task consists of looking at the ticked inside the car and writing fines in case there are some infringement or if the ticket is not visible. So, we can consider that the system of parking in these zones is inefficient and uncomfortable for users as well as guards. The proposed model will seek to overcome these difficulties and improve its efficiency.

The main goal of this research is to develop a system that facilitates the client and the guard tasks. Nowadays, the mobile device is an essential tool daily used. That's why a mobile model is implemented so that an user manages his payments, receive notification if any infringement has incurred or know the position of the car at any time. As well, the guard can access via his mobile device to the vehicle identification mechanisms.

This identification was developed by NFC technology and by image recognition. Although there are previous studies that try and solve this problem efficiently [1], there are still difficulties to recognize plates, such as image resolution, 
color, lighting or computing load. Some ways to partially overcome these problems are the use of infrared camera to know about the position of plates and easily read digits [2]. In our case, the camera position is always variable because it is a mobile device. This is a difficulty that provokes the system not to work properly, so we consider essential the research and improvement of the techniques proposed for license plate recognition using a mobile device, and the integration of this system into a multiagent system to facilitate the communication among the server, the device and the data provider among others.

The paper is structured as follows: Section 2 explains a brief review about agents, NFC technology and image recognition. Section 3 describes the system, integrated with agents and with OCR techniques. Finally, section 4 presents preliminary results of NFC and image identification, studying several algorithms and their results as well as conclusions and future improvements in the system.

\section{Background}

Although agents field was studied widely to develop this system [3] and the utility of MAS solving Ambient Intelligence problems [4 6], our efforts in the background was dedicated to identification techniques. In particular, we research about states of the art in NFC and plate recognition.

\section{$2.1 \quad$ NFC}

NFC is the acronym for the concept Near Field Communication. The NFC is a wireless communication interface with a limited working distance of about 10 $\mathrm{cm}$. The interface can operate in several modes. The modes differ if a device creates its own field of radio frequency $(\mathrm{RF})$ or a device gets the power from the $\mathrm{RF}$ field generated by another device.

In the field of research, there are several studies on the possibility of applying NFC technology for monitoring certain medical-related parameters [7], or work related to the welfare of the people in the field of ambient intelligence [8] or studies focused on the security vulnerabilities that the NFC protocol can present and possible solutions [9].

\subsection{Plate Recognising}

Although there are commercial software, ANPR is being extensively investigated [1, 2, 10]. Commercial systems can get higher success rates to $95 \%$ in controlled environments. In environments where lighting conditions are not controlled (e.g. by infrared cameras) or varies the orientation or foreshortening of the plate size, the success rate drops to $75 \%$.

In the ANPR we can distinguish two phases: 1) Location of the plate within the image, critical step for subsequent identification and 2) the recognition and validation of license plate characters. The methods used to locate the license plate within the image are varied. In any case, the result would be each character 
separately. Subsequently, the recognition of the segmented characters is often implemented using neural networks. The entrance of the networks may be a direct representation of the characters or indirect, in which the characters suffer from some prior processing [10]. Others opt for the correlation, just as in [11], although in this case the procedure is modified with certain heuristic rules to improve their hit rate.

\section{System Description}

As discussed above, the system to be built is based on agents [12]. Note that the most important part of our research is to manage the identification, as it is the place where the studies have focused on image recognition, studying OCR with other clustering algorithms and neural networks. We study the NFC identification protocol as well.

The first step in this section include descriptions of agent-based system, to focus after on one target system: identification of an environmental object. We have employed GORMAS [13], and as anticipated in section 3, PANGEA [14] has been our ultimate development platform. In developing the design of the virtual organization and establish the model is necessary to analyze the needs and expectations of potential users of the system. The result of this analysis will be the roles of the entities involved in the proposed system. In particular, the following roles were found:

- User. Represents the user or client system. In this case, the user will be vigilant or client. The agent will separate because different functionality depending on the role you acquire.

- Identify Vehicle: This role is responsible for carrying out the process ID of the vehicle, it can run various methods according to the input parameters.

- Informer: It is responsible for presenting system communications. You can carry out several notifications, depending on what role or agent communicates to him. This role allows the user to interact with the system, and is responsible for collecting the ratings that the user can perform.

- Locator: This process is responsible for locating certain objects in the environment, as the vehicle or the user.

- Infringements: Its responsibility is to manage the whole system of infringements that occur when a client does not have appropriate behavior.

- Communication: It is responsible for communication between the virtual organization of the central server and the organization. This communication results in the response of certain agents according to the input parameters from the server OV.

- Supplier: This role is an agent that accesses and stores all or most of the information needed to manage the actions that govern this system. This information can be extracted through services such as data collection system masCloud [15], or by objects in the environment such as NFC tag, images or information provided by user roles. 
- Supervisor: An agent that exercises this role will have overall control of the system. Analyze the structure and syntax of all messages in and out of the system.

A fully description of this techniques will be presents on the following sections.

\subsection{Car Identification Techniques}

Managing vehicle identification has been a central part of our research. In this process, various techniques have been explored and chosen the most adapted to the needs. We will explore each of them, commenting on the study process and the development.

The NFC recognition is one of the techniques that allowed us to recognize an object in the environment, in our case the vehicle. The system is design in order to recognize habitual vehicles, that is, vehicles registered in our system. Unregistered vehicles are identified by using image recognition.

In NFC, the goal is to communicate an active device (mobile device) with a passive device (NFC tag). The advantage of these tags is that require no maintenance or batteries, are activated by the electromagnetic field itself. Each vehicle has a unique number that identifies your car. Thus, it can be read the card with its own mobile and identify the vehicle.

Furthermore, to identify the vehicle by image research about different programs already in operation was performed, but also on the facilities that support the library OpenCV [16]. Currently, OpenCV implements a module to interact with Android more efficiently, but can use native library algorithms such as the algorithm of K-means [17] and Multilayer Perceptron neural networks, without significantly increasing the runtime. Therefore, we chose this as the main tool to analyze and process the image to extract the text.

The next step was to process the image to extract the basic features of it and be able to recognize the digits. Specifically, we were ahead 4 main stages:

1. Remove the plate

2. Extract each digit separately

3. Recognize each digit

4. Check the accuracy of the recognized plate

To remove the plate, the same composition was studied, considering only the license plates of cars (most of our clients). Its design is unique. On the left is wearing a blue strip with the symbol of the European Union and the letter E for Spain, and contains three letters ranging from BBB to ZZZ, and 4 digits ranging from 0000 to 9999 . This character set is the necessary key to the identification of each vehicle. Being a white plate with black letters, it facilitates to some extent the image processing. The purpose of preprocessing is to obtain a binarized image, where there are only black and white colors.

There are many techniques to improve image quality and convert it in black and white. In fact, many times the recognition algorithm success depends heavily on this pre-processing and extraction of digits. In this study we have tried various 
forms of image processing, though the best results have been given, as described below.

To minimize calculus within the mobile device, three steps are followed:

1. Perform a transformation of the color image to grayscale image

2. Eliminate certain noise in the image, using an smoothing technique contours [18].

3. Eliminate certain imperfections in the image, using some morphological transformation.

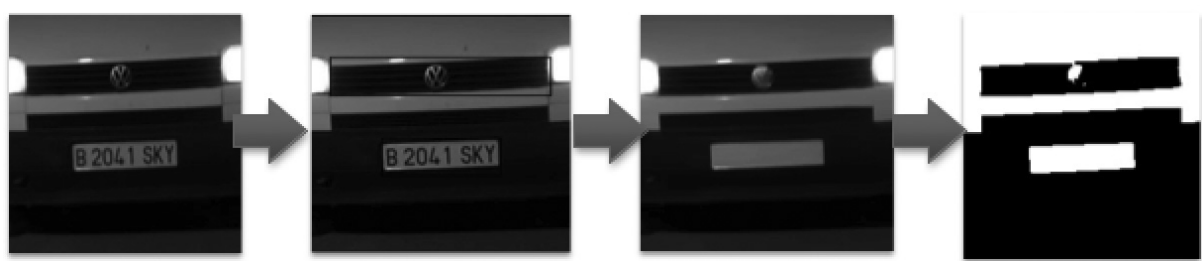

Fig. 1. Outlining the transformation of the image to identify the license plate

To extract the digits, a similar process was followed. Image 2 show the final result.

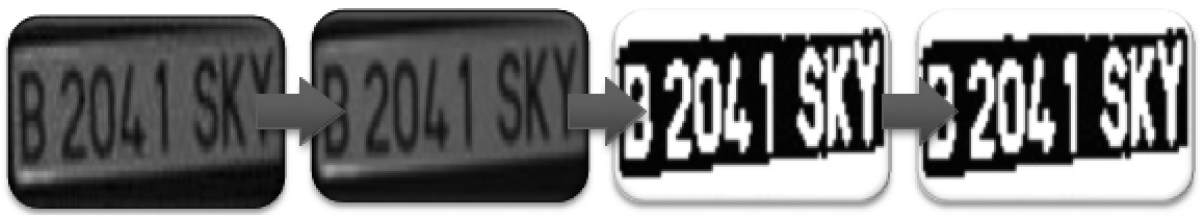

Fig. 2. Outlining the transformation of the image to identify the plate digits

To recognize the digit two techniques were studied. On one hand, various clustering algorithms are contemplated and finally opted for the study of the nearest neighbour algorithm. Furthermore, a multiperceptron neural network was trained, and the results were observed. In particular, we consider 50 neighbors for the clustering algorithm, and a multilayer perceptron net in the case of neural nets, with only one hidden layer of 150 neurons, 144 entries (one for each pixel of the image), and 28 output neurons, one for each letter. A tansig function is considered as the activation function in hidden layer, and a logsig function for output layer.

In both cases it has had to create a set of training data, and test data. For training, eight examples of each letter, considering that may have different inclinations (included in the training set) are created. With this training set, the 
neural network and the clustering algorithm was trained and then tested with images collected from real examples, to study what level of precision and total error committed.

\section{Preliminary Results and Conclusions}

The degree of accuracy in identifying the vehicle with NFC is always $100 \%$. Tests have been carried out with four types of NFC tags of different technologies, depending on the capacity ( $0^{\prime} 5 \mathrm{~K}, 1 \mathrm{~K}$ or $2 \mathrm{~K}$ ), always with the same results. Possible security issues have not been taken into account due to the brevity of the communication between the tag and the mobile device. Still, it is desirable to study new innovations to protect this type of communication. This study is already in future lines.

There were two difficulties when collecting data. The first was to develop an agent collecting data correctly by the necessary protocols. Fortunately, there are interfaces that facilitated the exchange of information between the active and passive device. The second difficulty was to study what was the maximum distance at which the mobile device detects the tag. This was an important functionally because the guard wants the best possible comfort to read labels. Therefore, it was investigated on different ways to increase the recognition distance. In the end, the conclusion was that we relied mainly on hardware and type of active device used.

Test were made to study the results with license plate recognition in an image, with special attention to the type of lighting and features, and the type of algorithm or structure implemented. The nearest neighbor algorithm was set to 50 neighbors to consider when classifying each character. Neural networks were a little more complex to define and train, so it was considered only a Multilayer Perceptron network with a hidden layer. The first problem was precisely determine the most appropriate network structure. From the outset, we consider a network with an input pixel. It was determined that each image must be 12 pixels high by 12 pixels wide, which left us with a neural network of 144 entries. The number of outputs had to be equal to the total points ( 28 output neurons, one for each letter).

After several tests, the number of neurons in the hidden layer 150, with a tangential activation function (the most commonly used to recognize characters [19]) was fixed.

We present an illustrative table below (Table1) with the results obtained using the nearest neighbor algorithm, compared with neural networks. The success rate is also presented in recognizing the license plate and each of the digits. The data have been calculated at 25 test plate images.

Upon closer analysis, it was revealed that some characters such as $\mathrm{M}$ or $\mathrm{W}$ are recognized in most cases, while others were easily confused with each other. Still, the error level increased if tested were distorted or in low resolution. In particular, we note that the letter $\mathrm{B}$ was often recognized as the number 8 , the $\mathrm{G}$ and the number 6 . This confusion between numbers and letters could be 
Table 1. Comparative table of results at each stage of recognition

\begin{tabular}{|c|c|c|}
\hline Stage & Clustering & Neural Nets \\
\hline Plate Defin & \multicolumn{2}{|c|}{$84 \%(21 / 25)$} \\
\hline Digits Defining & \multicolumn{2}{|c|}{$865 \%(173 / 200)$} \\
\hline \multicolumn{3}{|c|}{ Characters Recognition 60\% (120/200) 80'5\% (161/200) } \\
\hline
\end{tabular}

overcome by introducing a condition. If the recognized digit was one of the first four, would be recognized as a letter. Otherwise, be recognized as a number. Thus, if for example, detect an 8 on the second digit, we would consider a B Of course, an improvement of this solution is possible if the recognizer is improved with some fuzzy logic patterns or more training sets.

Moreover, some characters with similar patterns are not correctly recognized. For example, X, K and Y, or B and G. For the latter problem the only plausible solution was to fatten the number of training patterns, and improve the recognizer.

The execution times of license plate recognition depended on the different characteristics of the image. Still, we can set the average execution time of about 4 seconds. It is an acceptable time, if a little slow for the mobile environment in which it is developed. Improving this time and the results shown is considered as future lines.

Another highlight was the successful development of virtual organizations to achieve the objectives of this work. The process of identifying and organizing roles helped improve the management and thus to improve efficiency. PANGEA as platform allowed fluid communication between agents, which is evident in the design of the application.

\section{Conclusions}

The research presented herein aims to bring new perspectives in the field of image recognition using the multiagent systems to communicate data server and camera from a mobile device. Also research in the field of virtual organizations was essential for the proper management of the implementation of a multiagent system. In this regard, it has been studied and used a development-oriented organization method that covers the analysis and design of the structure of de virtual organization, showing that the system is feasible to solve our problem of parking automation.

With this approach, the expected benefits are to improve the users' comfort. On one hand, the user is able to safe his money, because he only pays the time he was parked. On the other hand, the payment, the payment renovation after the limit of time imposed by the enterprise is made only touching a button. Also, the model assists the guards by identifying the vehicle in a very easy way, only aproximating the mobile device to the stick in the car glass. This might 
provoke that the enterprise can have less employeers. Also, the enterprise can take advantage of the users' vehicle localization to advertise restaurants or shops near the user.

So, could you discuss the expected profits estimation as well as possibility of direct application of the results provided. What about a real-life verification of the approach proposed?

We have shown that there is a viable way of recognizing digits using a camera of a mobile device. However, the results have not been as good as expected at first. As a future line, we propose to improve results not only on accuracy, but at runtime, essential because of the limited resources of the mobile device and the nature of it (easy to use and fast). Some proposals to reduce this time could be to use another clustering algorithm, another neural network structure or even change the network. This should never be detrimental to the accuracy of the results. Anyway, it is viable to use this identification system in a real system, allowing the guard to correct manually if there is a mistake in the plate recognition.

In addition to these improvements, this application can be analyzed for future research in other fields of research. For example, the application could tell the user what places are free, looking for a new algorithm fuzzy logic to decide in terms of traffic and a number of certain parameters. However, this work has not been explored yet.

Acknowledgements. This work has been partially supported by the Spanish Government through the project iHAS (grant TIN2012-36586-C01/C02/C03).

\section{References}

1. López Fernández, J.M.: Software para el reconocimiento automático de matrículas (2006)

2. Vázquez, N., Nakano, M., Pérez-Meana, H.: Automatic system for localization and recognition of vehicle plate numbers. Journal of Applied Research and Technology (2002)

3. Rodrguez, S., de Paz, Y., Bajo, J., Corchado, J.: Social-based planning model for multiagent systems. Expert Systems with Applications 10(38), 13005-13023 (2010)

4. Corchado, J., Fyfe, C.: Unsupervised neural method for temperature forecasting. Artificial Intelligence in Engineering 4(13), 351-357 (1999)

5. Tapia, D., Abraham, A., Corchado, J., Alonso, R.: Agents and ambient intelligence: case studies. Journal of Ambient Intelligence and Humanized Computing 2(1), 85$93(2010)$

6. Fdez-Riverola, F., Corchado, J.: Cbr based system for forecasting red tides. Knowledge-Based Systems 5(16), 321-328 (2003)

7. Patrick, K., Griswold, G., Raab, F., Intille, S.S.: Health and the mobile phone. Technical report (2008)

8. Morak, J., Kumpusch, H., Hayn, D., Modre-Osprian, R., Schreier, G.: Design and evaluation of a telemonitoring concept based on nfc. Enabled Mobile Phones and Sensor Devices. IEEE Transactions on Information Technology in Biomedicine 
9. Haselsteiner, E., Breitfuss, K.: Security in near field communication. strengths and weakness. Technical report (2012)

10. Shyang-Lih, C., Sei-Wan, C.: Automatic license plate recognition. IEEE Trans. on Intelligent Transportation Systems

11. Martín, F., Borges, D.: Automatic car plate recognition using partial segmentation algorithm. In: SPPRA

12. Venturini, V., Carbo, J., Molina, J.M.: Methodological design and comparative evaluation of a mas providing ami. Expert Systems with Applications 39(12), 10656-10673 (2012)

13. Argente, E., J. Botti, I.V.: GORMAS: Guía para el desarrollo de sistemas multiagente abiertos basados en organizaciones. PhD thesis (2008)

14. Zato, C., et al.: PANGEA - Platform for Automatic coNstruction of orGanizations of intElligent Agents. In: Omatu, S., Paz Santana, J.F., González, S.R., Molina, J.M., Bernardos, A.M., Rodríguez, J.M.C. (eds.) Distributed Computing and Artificial Intelligence. AISC, vol. 151, pp. 229-239. Springer, Heidelberg (2012)

15. De la Prieta, F., Rodríguez, S., Bajo, J., Corchado, J.M.: A Multiagent System for Resource Distribution into a Cloud Computing Environment. In: Demazeau, Y., Ishida, T., Corchado, J.M., Bajo, J. (eds.) PAAMS 2013. LNCS (LNAI), vol. 7879, pp. 37-48. Springer, Heidelberg (2013)

16. Bradski, G., Kaehler, A.: Computer vision with the opencv library. Technical report (2008)

17. Dhillon, I.S., Guan, Y., Kulis, B.: Kernel k-means: spectral clustering and normalized cuts. In: Proceedings of the Tenth ACM SIGKDD International Conference on Knowledge Discovery and Data Mining, pp. 551-556. ACM (2004)

18. Wahl, F.M.: Image preprocessing procedure for noise removal US Patent 4,747,156 (May 24, 1988)

19. Holley, R.: How good can it get? analysing and improving ocr accuracy in large scale historic newspaper digitisation programs. D-Lib Magazine 15(3/4) (2009) 